\section{Research kudos does not need a price tag}

We are concerned that the focus on generating grant income to fill university coffers penalizes science that is good value for money. As research money dwindles, the winning of funds seems to be emerging as the way to judge performance. This criterion is used by the UK universities' Research Excellence Framework, for example, and to assess researchers for hiring or promotion.

Huge strides are being made in our field of wholeorganism biology owing to large collaborative grants that help to pay for salaries and equipment. However, important oases of the biological sciences are relatively unaffected by the benefits of big grants. Examples include biological modelling, ecological projects in the developing world and meta-analyses based on literature mining.

At a time when economic efficiency is paramount in publicly funded areas such as health and education, an undue emphasis on generating money could drive scientists to compete for limited public funds simply for career purposes. In our view, funding success must not become a disproportionate factor in gauging scientific achievement. Tim Caro University of California, Davis, USA. Sasha R. X. Dall University of Exeter, Penryn, Cornwall, UK. tmcaro@ucdavis.edu

\section{Don't misrepresent bats' link with SARS}

We find your report on bats and severe acute respiratory syndrome (SARS) sensationalist and misleading (Nature 552, 15-16, 2017). The important work it discusses does not claim to pinpoint conclusively the source of the SARS outbreak (B. Hu et al. PLoS Pathog. 13, e1006698; 2017), as implied by your "smoking gun" metaphor.
The rapid rate of evolution of RNA viruses means that SARS could have arisen in one of many areas. Thus, your inference that the strain "could easily" have originated in this bat population is, in our view, unjustified.

Inflammatory statements about bats and disease have led to culling and roost destruction, compromising conservation efforts (K. J. Olival EcoHealth 13, $6-8 ; 2016)$. Accurate reporting of information on SARS, Middle East respiratory syndrome, Ebola and other emerging diseases is crucial for controlling outbreaks and for preventing unnecessary deaths of wild animals.

Viral spillover occurs when humans and domestic animals come into direct contact with wild animals and their pathogens. Public education, comprehensive surveillance and considered interventions can all help to protect public health. The closure of markets selling live birds has already reduced the activity of avian influenza viruses, and could likewise curtail the spillover of mammalian viruses.

Paul A. Racey ${ }^{\star}$ University of Exeter, Penryn, Cornwall, UK. p.a.racey@exeter.ac.uk ${ }^{\star}$ On behalf of 5 correspondents (see go.nature.com/2qzjxt4 for full list).

\section{Train robots to self-certify as safe}

Robots can operate autonomously in extreme environments that might be hazardous for humans. For example, they can inspect oil and gas equipment, monitor offshore wind turbines, survey subsea power networks and maintain nuclear reactors. We suggest that these robots should be required to self-certify that they can operate safely under such circumstances.

Robots can learn to adapt the way they perform tasks in changing and unexpected environments. However, if a robotic system learns a flawed model of the environment or a risky behaviour, it could undermine its own operation and the integrity of the asset that it is inspecting or repairing - with potentially catastrophic consequences. To protect against this, the robot should self-certify its correct operation by collecting data as it executes its task. It would then check the data against its mission plan, with minimal input from human operators (see D. M. Lane et al. IFAC Proc. Vol. 45, 268-273; 2012).

For autonomous systems to be trusted, developments in robotics and artificial intelligence need to be accompanied by advances in certification techniques. Regulators such as Lloyd's Register have certification standards for industrial equipment and are beginning to explore the challenges of certifying selflearning systems (see go.nature. com/2cxxjcx). And several teams at Research Councils UK, including the Offshore Robotics for Certification of Assets hub (https://orcahub.org), are investigating this crucial area. Valentin Robu, David Flynn, David Lane Heriot-Watt University, Edinburgh, UK. v.robu@hw.ac.uk

\section{Statistics: a social and cultural issue}

Too many practitioners who discuss the misuse of statistics in science propose technical remedies to a problem that is essentially social, cultural and ethical (see J. Leek et al. Nature 551, 557-559; 2017). In our view, technical fixes are doomed.

As Steven Goodman writes in the article, there is nothing technically wrong with $P$ values. But even when they are correct and appropriate, they can be misunderstood, misrepresented and misused - often in the haste to serve publication and career. $P$ values should instead serve as a check on the quality of evidence.

The great paradox of science is that passionate practitioners must carefully produce dispassionate facts (J. Ravetz
Scientific Knowledge and its Social Problems Oxford Univ. Press; 1971). Meticulous technical and normative judgement, as well as morals and morale, are necessary to navigate the forking paths of the statistical garden.

Unless peer review and rewards in academia change to encourage such virtues, the present crisis will remain intractable (see also A. Saltelli and S. Funtowicz

Futures 91, 5-11; 2017). Andrea Saltelli University of Bergen, Norway. Philip Stark University of California, Berkeley, USA. andrea.saltelli@uib.no

\section{Statistics: deploy with integrity}

Discussions to strengthen the quality of statistical analyses are a welcome demonstration of scientists' willingness to confront uncomfortable knowledge (J. Leek et al. Nature 551, 557-559; 2017). Just as science in general is not a truth machine, statistics is not a device for automatically bootstrapping certainty out of data sets.

All users of statistical techniques, as well as those in other mathematical fields such as modelling and algorithms, need an effective societal commitment to the maintenance of quality and integrity in their work. If imposed alone, technical or administrative solutions will only breed manipulation and evasion.

There may be methodological issues as well. For example, we are only now discovering that the universally accepted standard tests, notably significance and $P$ values, are simplistic and misleading. It might be that improved tests, such as those involving power calculations, are just too sophisticated for otherwise competent researchers. If so, then the conduct of empirical science will need substantial modification. Jerome Ravetz University of Oxford, UK.

jerome.ravetz@gmail.com 